\title{
The system of four reggeized gluons and the three-pomeron vertex in the high colour limit
}

\author{
M. Braun \\ Dep. High-Energy Physics, University of St. Petersburg, \\ 198904 St.Petersburg, Russia
}

May 1997

\begin{abstract}
.
The amplitude for 4 interacting reggeized gluons is studied in the high-colour limit. The leading order amplitude is explicitly shown to reduce to a pair of reggeons, i.e to a single BFKL pomeron. The next-to-leading order diffractive amplitude is found to split into a double pomeron exchange and triple pomeron contribution. The obtained three-pomeron vertex is different from the originally proposed in $[2]$.
\end{abstract}

SPbU-97-9 


\section{Introduction.}

The system of four reggeized gluons has been studied in detail by J.Bartels [1] and J.Bartels and M.Wuesthoff [2] for the realistic case of the number of colours $N=3$. The results are quite complicated because of the interconnection between many different colour channels. The amplitudes obey a system of coupled integral equations for which the inhomogeneous term is provided by a part which is supposed to reduce to a pair of reggeons (the "reggeizing piece") coupled to the non-reggeizing piece by a transitional vertex. This vertex presents some nice features: it is symmetric in all the gluons, infrared stable, conformal invariant [3] and vanishes if any of the extrnal momenta goes to zero. However its definition is rather dubious: in fact to obtain it in the form presented in [2] it was assumed that a pair of reggeized gluons may combine into a single reggeized gluon not only in the adjoint representation but also in other ones, which does not agree with the corresponding BFKL equation.

In this note we study the same problem under the simplifying assumption $N \rightarrow \infty$. As we already stated in [4], in the leading approximation, the amplitude for any number of reggeized gluons reduces to the one for only a pair of them, i.e to a BFKL pomeron. In the language of $[1,2]$ this means that the non-reggeizing piece is down by at least $1 / N$ as compared to the reggeizing piece for the leading colour configuration (a cylinder in the picture in which the gluon is represented by a $q \bar{q}$ line). We demonstrate this fact in some detail for the triple discontinuity of the 4-gluon amplitude in Sec. 2 .

As a result, a possibility opens up to study the complicated Bartels system for different colour channel amplitudes by perturbation in $1 / N$ starting from the fully reggeizing piece in the leading approximation. In this manner one arrives at a uniquely defined transitional vertex which is different from [2], although it preserves some of its good features. In particular, it is also infrared stable and vanishes for any of the external momenta going to zero. Apart from this, a new contribution arises which corresponds to a direct coupling of two pomerons to a $q \bar{q}$ loop. It has an eikonal form for fixed transversal dimension of the loop. This fact was also conjectured in [4]. Thus the $N \rightarrow \infty$ approach allows to uniquely separate contributions from the double pomeron exchange and the triple pomeron coupling, which remain hidden in the transitional vertex of [2].

\section{The 4 gluon system at $N \rightarrow \infty$}

\subsection{The 2 gluon system}

The results for 2 gluons are well-known and are not sensitive for the $N \rightarrow \infty$ limit. We only list here the main formulas to be used in the following. In our notations we try to follow $[1,2]$.

In the lowest (zero) order approximation the 2 gluon amplitude (actually its discontinuity Mellin transformed to the complex angular momentum $j$ ) is given by the $q \bar{q}$ loop with the two gluons attached to it in all possible ways (4 diagrams in all). Their colour indeces $a_{1}$ and $a_{2}$ enter into the colour trace $\operatorname{Tr}\left\{t_{a_{1}} t_{a_{2}}\right\}=(1 / 2) \delta_{a_{1} a_{2}}$, where $t_{a}$ is the colour of the quark.. Separating this trace and the coupling $g^{2}$ we write the zero order contribution as

$$
D_{20}\left(k_{1}, a_{1}, k_{2}, a_{2}\right)=(1 / 2) \delta_{a_{1} a_{2}} g^{2}\left(f\left(0, k_{1}+k_{2}\right)+f\left(k_{1}+k_{2}, 0\right)-f\left(k_{1}, k_{2}\right)-f\left(k_{2}, k_{1}\right)\right)
$$

Here $f\left(k_{1}, k_{2}\right)$ is the contribution from the diagram with the gluon 1 attached to $q$ and the gluon 2 
attached to $\bar{q}$. Assuming that the external particle is colourless (the photon or "onium") we have $f\left(k_{1}, k_{2}\right)=f\left(k_{2}, k_{1}\right)$. We define a vacuum colour state of two gluons by its colour wave function

$$
|0\rangle=(1 / N) \delta_{a_{1} a_{2}}
$$

Then we find a projection of the $q \bar{q}$ loop onto this state from (1)

$$
\left\langle D_{20} \mid 0\right\rangle=\frac{N^{2}-1}{N} g^{2}\left(f(0,0)-f\left(k_{1}, k_{2}\right)\right) \simeq g^{2} N\left(f(0,0)-f\left(k_{1}, k_{2}\right)\right) \equiv D_{20}(1)
$$

In the last notation, to be extensively used in the following, only the number of the gluon whose momentum acts as one of the variables is indicated, the other momentum determined from the conservation law (e.g. $k_{2}=-k_{1}$ for the forward amplitude).

The full 2-gluon amplitude $D_{2}$ satisfies the vacuum channel BFKL equation

$$
S_{20} D_{2}=D_{20}+g^{2} N V_{12} D_{2}
$$

where $S_{20}$ is the 2 gluon "free" Schroedinger operator for the energy $1-j$

$$
S_{20}=j-1-\omega(1)-\omega(2)
$$

$\omega(k)$ is the gluon Regge trajectory (see e.g [5]) and $V_{12}$ is the BFKL interaction with the kernel

$$
V\left(1,2 ; 1^{\prime}, 2^{\prime}\right)=\frac{k_{1}^{2}{k_{2}^{\prime}}^{2}+k_{2}^{2}{k_{1}^{\prime}}^{2}}{{k_{1}^{\prime}}^{2}{k_{2}^{\prime}}^{2}\left(k_{1}-k_{1}^{\prime}\right)^{2}}-\frac{\left(k_{1}+k_{2}\right)^{2}}{{k_{1}^{\prime 2} k_{2}^{\prime 2}}^{2}}
$$

Our aim in this section is to demonstrate that, to the leading order in $N$, the 4 gluon amplitude is reduced to the 2 gluon amplitude $D_{2}$, which is asumed to be known as the solution to (4). However before we go to 4 gluons we have to study the 3 gluon case.

\subsection{The 3 gluon system}

Still at this level no simplifications occur in the high-colour limit, so that the results can be borrowed from $[1,2]$. Three gluons may combine either into a fully symmetric colourless state ( $d$-coupling) or into a fully antisymmetric one ( $f$-coupling). In the high colour limit it is more convenient to use their combination

$$
h_{a_{1} a_{2} a_{3}}=d_{a_{1} a_{2} a_{3}}+i f_{a_{1} a_{2} a_{3}}
$$

cyclic symmetric in the gluons 1,2 and 3 and with the properties

$$
\begin{gathered}
h_{a_{1} a_{2} a_{3}}^{*}=h_{a_{2} a_{1} a_{3}} \\
\sum_{c d} h_{a c d}^{*} h_{b c d}=\delta_{a b} 2 N\left(1-2 / N^{2}\right) \\
\sum_{c d} h_{a c d} h_{b c d}=-\delta_{a b}(4 / N)
\end{gathered}
$$

Normalizing, we introduce two colour wave functions

$$
|123\rangle=\frac{1}{\sqrt{2 N^{3}}} h_{a_{1} a_{2} a_{3}}, \quad|213\rangle=\frac{1}{\sqrt{2 N^{3}}} h_{a_{2} a_{1} a_{3}}
$$


which are orthogonal in the limit $N \rightarrow \infty$, due to (8). They correspond to the three gluons lying on the surface of a cylinder in a sequence 123 or 213 in the direction of the quark loop. Since each pair of the gluons is in the adjoint representation, the interaction will not change the colour configuration. So the equations for the 3 gluon amplitude in the two colour states $|123\rangle$ and $|213\rangle$ decouple.

To set up these equations we need their zero-order terms. They are provided by the quark loop with 3 gluons attached to it in all possible ways ( 8 diagrams). The colour factor is given by the trace

$$
\operatorname{Tr}\left\{t_{a_{1}} t_{a_{2}} t_{a_{3}}\right\}=(1 / 8) h_{a_{1} a_{2} a_{3}}
$$

if the gluons run in the order 123 along the quark line. The rest of the contribution is given by the same function $f$ as in (1), in which the momenta of the gluons attached to the same line (i.e the quark line or the antiquark line) sum together and the overall sign is given by $(-1)^{n_{q}}$ where $n_{q}$ is the number of gluons attached to the quark line. Projecting onto the states (9) we find

$$
D_{30}^{(123)}=-D_{30}^{(213)}=g \sqrt{N / 8}\left(D_{20}(2)-D_{20}(1)-D_{20}(3)\right)
$$

In the 3 gluon equation, apart from the inhomogeneous term (10), an aditional contribution appears coming from possible transitions from 2 to 3 gluons (Fig. 1). These transitions are accomplished by the vertex $K_{2 \rightarrow 3}$ introduced by J.Bartels [6]:

$$
K_{2 \rightarrow 3}=i g^{3} f^{a_{1}^{\prime} a_{1} c} f^{c a_{2} d} f^{d a_{3} a_{3}^{\prime}} W\left(1,2,3 ; 1^{\prime} 3^{\prime}\right)
$$

for the transition of two gluons $\left(k_{1}^{\prime}, a_{1}^{\prime}\right)$ and $\left(k_{3}^{\prime}, a_{3}^{\prime}\right)$ into three gluons $\left(k_{1}, a_{1}\right),\left(k_{2}, a_{2}\right)$ and $\left(k_{3}, a_{3}\right)$. The kernel $W$ is symmetric in the gluons 1 and 3 and can be expresed through the BFKL kernel as

$$
W\left(1,2,3 ; 1^{\prime} 3^{\prime}\right)=V\left(2,3 ; 1^{\prime}-1,3^{\prime}\right)-V\left(1+2,3 ; 1^{\prime} 3^{\prime}\right)
$$

In the following one of the pairs of final gluons 12 or 23 will have a definite total colour. As a result the prefactor in (11) can be simplified to

$$
i g^{3} T^{(12)} \delta_{a_{1} a_{1}^{\prime}} f^{a_{2} a_{3} a_{3}^{\prime}}
$$

or to

$$
i g^{3} T^{(23)} \delta_{a_{3} a_{3}^{\prime}} f^{a_{1}^{\prime} a_{1} a_{2}^{\prime}}
$$

for the two mentioned cases, respectively, where $T$ is the total colour in each case ( $-N$ for the colourless state and $-N / 2$ for the adjoint state). Projecting onto the state $|123\rangle$ we find a contribution

$$
D_{2 \rightarrow 3}^{(123)}=g^{3} \sqrt{N^{3} / 8} W\left(1,2,3 ; 1^{\prime} 3^{\prime}\right) \otimes D_{2}\left(1^{\prime}, 3^{\prime}\right) \equiv g^{3} \sqrt{N^{3} / 8} W_{2}(1,2,3)
$$

Here the symbol $\otimes$ means integration over primed variables with the weight $(2 \pi)^{-3} \delta^{2}\left(1+2+3-1^{\prime}-3^{\prime}\right)$. This integration and the function $D_{2}\left(1^{\prime}, 3^{\prime}\right)$ are implicit in the abbreviated notation in the second equality (the subindex 2 shows that it is the function $D_{2}$ which should be integrated with the kernel $W)$.

The complete equation for the $|123\rangle$ colour state is thus

$$
S_{30} D_{3}^{(123)}=D_{30}^{(123)}+D_{2 \rightarrow 3}^{(123)}+(1 / 2) g^{2} N\left(V_{12}+V_{23}+V_{31}\right) D_{3}
$$


The operator $S_{30}$ is a natural generalization of (5) to 3 gluons. The factor $1 / 2$ in front of the interaction part is due to the fact that all three gluons are now in the adjoint representation together with their neighbours.

The equation for the $|213\rangle$ state has opposite signs for its inhomogeneous parts. Therefore we find

$$
D_{3}^{(213)}=-D_{3}^{(123)}
$$

The seemingly complicated equation (14) can however be easily solved. As shown in [1] its full solution is simply the zero-order term with all $D_{20}$ substituted by the BFKL pomerons $D_{2}$ ("the reggeized zero order term"):

$$
D_{3}^{(123)}=-D_{3}^{(213)}=g \sqrt{N / 8}\left(D_{2}(2)-D_{2}(1)-D_{2}(3)\right)
$$

This fact can be demonstrated by a direct substitution. We shall derive it in a different manner, using the idea that a pair of reggeized gluons in the adjoint representation combines in a single reggeized gluon, presented in [4]. This allows to easily generalize the derivation to the 4 gluon case.

Consider a 3 gluon equation with a zero-order term $D_{2}(1)$

$$
S_{30} D_{3}^{(1)}=D_{20}(1)+F_{3}^{(1)}+(1 / 2) g^{2} N\left(V_{12}+V_{23}+V_{31}\right) D_{3}^{(1)}
$$

with a certain additional inhomogeneous term $F_{3}^{(1)}$. As shown in [4], the solution to (17) will be given by

$$
D_{3}^{(1)}=D_{2}(1)
$$

provided we choose

$$
F_{3}^{(1)}=-(1 / 2) g^{2} N\left(W_{2}(2,3,1)+W_{2}(3,2,1)\right)
$$

(see definition (13)).

Indeed, put (18) into Eq. (17). The interaction $V_{23}$ gives

$$
(1 / 2) g^{2} N V_{23} D_{2}(1)=(\omega(2+3)-\omega(2)-\omega(3)) D_{2}(1)
$$

("the bootstrap relation", [7]) and thus converts the operator $S_{30}(1,2,3)$ into $S_{20}(1,2+3)$. The two other interaction terms give explicitly

$$
(1 / 2) g^{2} N\left(V\left(1,2 ; 1^{\prime}, 2^{\prime}\right) \otimes D_{2}\left(1^{\prime}, 2^{\prime}+3\right)+V\left(3,1 ; 3^{\prime}, 1^{\prime}\right) \otimes D_{2}\left(1^{\prime}, 2+3^{\prime}\right)\right)
$$

On the other hand, using (12), the term $F_{3}^{(1)}$ has the form

$$
\begin{aligned}
F_{3}^{(1)}= & -(1 / 2) g^{2} N\left(V\left(3,1 ; 2^{\prime}-2,1^{\prime}\right) \otimes D_{2}\left(2^{\prime}, 1^{\prime}\right)-V\left(2+3,1 ; 2^{\prime}, 1^{\prime}\right) \otimes D_{2}\left(2^{\prime}, 1^{\prime}\right)\right. \\
& \left.+V\left(2,1 ; 3^{\prime}-3,1^{\prime}\right) \otimes D_{2}\left(3^{\prime}, 1^{\prime}\right)-V\left(3+2,1 ; 3^{\prime}, 1^{\prime}\right) \otimes D_{2}\left(3^{\prime}, 1^{\prime}\right)\right)
\end{aligned}
$$

Taking as integration variables $3^{\prime}=2^{\prime}-2$ in the first term in (21) and $2^{\prime}=3^{\prime}-3$ in the third, we find that these terms cancel the second and first terms in (20), respectively. The second and fourth terms in (21) give in the sum

$$
g^{2} N V\left(1,2+3 ; 1^{\prime}, 2^{\prime}\right) \otimes D_{2}\left(1^{\prime}, 2^{\prime}\right)
$$


so that the equation (17) becomes

$$
S_{20}(1,2+3) D_{2}(1,2+3)=D_{20}(1,2+3)+g^{2} N V_{1,2+3} D_{2}(1,2+3)
$$

which is evidently true.

The described procedure corresponds to combining two reggeized gluons 2 and 3 in the adjoint representation into a single reggeized gluon with the momentum $2+3$. For it to work, it is evidently necessary that the zero order term be a function of only the sum $2+3$ and also that a certain inhomogeneous term (19) be present which describes the necessary contributions from the transitions from 2 to 3 gluons.

Now we repeat this exercise with zero-order terms $D_{20}(2)$ and $D_{20}(3)$ and find two more solutions $D_{3}^{(2)}=D_{2}(2)$ and $D_{3}^{(3)}=D_{2}(3)$ which require additional inhomogeneous terms $F_{3}^{(2)}$ and $F_{3}^{(3)}$, respectively, obtained from (19) by permutations of 123 to 231 and 312. Taking the combination (16) of these solutions we find that the additional inhomogeneous term to be added is

$$
g \sqrt{N / 8}\left(F_{3}^{(2)}-F_{3}^{(1)}-F_{3}^{(3)}\right)=g^{3} \sqrt{N^{3} / 8} W_{2}(1,2,3)
$$

which is precisely the term we have in Eq. (14) for the 3-gluon amplitude. Therefore (16) is indeed its solution.

\subsection{The 4-gluon system}

On the 4 gluon level simplifications due to $N \rightarrow \infty$ become essential. At zero-order the 4 gluon amplitude is given by the quark loop with 4 gluons attached to it in all possible ways (16 diagrams). For a given order of gluons along the quark line, say, 1234, the cyclic symmetric colour trace is

$$
\operatorname{Tr}\left\{t_{a_{1}} t_{a_{2}} t_{a_{3}} t_{a_{4}}\right\}=(1 / 8) h_{a_{1} a_{2} b} h_{a_{3} a_{4} b}+(1 / 4 N) \delta_{a_{1} a_{2}} \delta_{a_{3} a_{4}}
$$

At $N \rightarrow \infty$ we neglect the second term and the sum $h_{a_{1} a_{2} b} h_{a_{3} a_{4} b}$ becomes cyclic symmetric in the gluons. It defines a colour state of 4 gluons

$$
|1234\rangle=\frac{1}{2 N^{2}} h_{a_{1} a_{2} b} h_{a_{3} a_{4} b}
$$

corresponding to the gluons lying on the surface of a cylinder in the order 1234 and in the adjoint representation with their neighbours. Other states are obtained from this by permutations of 234 . In the limit $N \rightarrow \infty$ they are orthonormalized. Projecting the quark loop onto these states we find

$$
\begin{gathered}
D_{40}^{(1234)}=D_{20}^{(4321)}=(1 / 4) g^{2} N\left(D_{20}(1)+D_{20}(4)-D_{20}(1+4)\right) \\
D_{40}^{(2134)}=D_{20}^{(4312)}=(1 / 4) g^{2} N\left(D_{20}(2)+D_{20}(3)-D_{20}(1+2)-D_{20}(1+3)\right)
\end{gathered}
$$

Interactions between the gluons do not change their overall colour state. Indeed, interactions between neighbour gluons are diagonal in colour since neighbours are all in the adjoint representation. Interactions across the cylinder (e.g. 13 in the state $|1234\rangle$ ) are down by $1 / N$ and can be neglected in the leading approximation. Therefore at $N \rightarrow \infty$ the complicated Bartels system for the 4 gluon amplitudes reduces to 4 decoupled equations for colour configurations appearing in (24) and (25). 
Moreover, one finds that the amplitudes with the inverse order of gluons (e.g. 1234 and 4321) are equal. As a result we arrive at only two different equations for colour states $|1234\rangle$ and $|2134\rangle$.

To set up these equations we need to find contributions from transitions into the 4 gluon state from 2- and 3-gluon states. The transition from 2 to 4 gluons corresponds to a single diagram, shown in Fig. 2. It is accomplished by a vertex similar to (11)

$$
K_{2 \rightarrow 4}=-g^{2} f^{a_{1}^{\prime} a_{1} c} f^{c a_{2} d} f^{d a_{3} e} f^{e a_{4} a_{4}^{\prime}} W\left(1,2+3,4 ; 1^{\prime}, 4^{\prime}\right)
$$

If gluons 12 and 34 have a definite total colour $T$ the prefactor in (26) simpljfies to

$$
-g^{4} T^{(12)} T^{(34)} \delta_{a_{1} a_{1}^{\prime}} \delta_{a_{2} a_{3}} \delta_{a_{4} a_{4}^{\prime}}
$$

For the amplitudes in the states indicated in (24) and (25) we have $T^{(12)}=T^{(34)}=-N / 2$. Projecting (26) onto these states we obtain the contribution to the state $|1234\rangle$ (and $|4321\rangle$ )

$$
D_{2 \rightarrow 4}^{(1234)}=-(1 / 4) g^{4} N^{2} W_{2}(1,2+3,4)
$$

The contribution from the transition 2 to 4 to the states $|2134\rangle$ and $|4312\rangle$ turns out to be zero.

Transitions from 3 to 4 gluons are described by four diagrams shown in Fig. 3 and are accomplished by the vertex $K_{2 \rightarrow 3}$ described above. Projecting onto the states $|1234\rangle$ and $|2134\rangle$ we find the following contributions. For $|1234\rangle$

$$
D_{3 \rightarrow 4}^{(1234)}=g^{3} \sqrt{N^{3} / 8}\left(W\left(2,3,4 ; 2^{\prime}, 4^{\prime}\right) \otimes D_{3}^{(124)}\left(1,2^{\prime}, 4^{\prime}\right)+W\left(1,2,3 ; 1^{\prime}, 3^{\prime}\right) \otimes D_{3}^{(134)}\left(1^{\prime}, 3^{\prime}, 4\right)\right)
$$

and for $|2134\rangle$

$$
D_{3 \rightarrow 4}^{(2134)}=-g^{3} \sqrt{N^{3} / 8}\left(W\left(1,2,4 ; 1^{\prime}, 4^{\prime}\right) \otimes D_{3}^{(134)}\left(1^{\prime}, 3,4^{\prime}\right)+W\left(1,3,4 ; 1^{\prime}, 4^{\prime}\right) \otimes D_{3}^{(124)}\left(1^{\prime}, 2,4^{\prime}\right)\right)
$$

Thus we find the equations for the two independent 4 gluon amplitudes in the form

$$
S_{40} D_{4}^{(1234)}=D_{40}^{(1234)}+D_{2 \rightarrow 4}^{(1234)}+D_{3 \rightarrow 4}^{(1234)}+(1 / 2) g^{2} N\left(V_{12}+V_{23}+V_{34}+V_{41}\right) D_{4}^{(1234)}
$$

and

$$
S_{40} D_{4}^{(2134)}=D_{40}^{(2134)}+D_{3 \rightarrow 4}^{(2134)}+(1 / 2) g^{2} N\left(V_{21}+V_{13}+V_{34}+V_{42}\right) D_{4}^{(2134)}
$$

We are going to demonstrate that solutions to these equations are exactly given by their zero-order terms $D_{40}$ in which $D_{20}$ are substituted by the BFKL pomerons $D_{2}$, that is

$$
D_{4}^{(1234)}=(1 / 4) g^{2} N\left(D_{2}(1)+D_{2}(4)-D_{2}(1+4)\right)
$$

and

$$
D_{4}^{(2134)}=(1 / 4) g^{2} N\left(D_{2}(2)+D_{2}(3)-D_{2}(1+2)-D_{2}(1+3)\right)
$$

To do it we apply the same trick as used in the 3 gluon case. Namely we shall find inhomogeneous terms necessary to combine 4 gluons into 2 for each term in the zero order expressions (24) and (25) separately. Then we shall sum the thus constructed pomerons into the combinations (32) and (33) and check that the resulting inhomogeneous terms will coincide with the terms $D_{2 \rightarrow 4}$ and $D_{3 \rightarrow 4}$ in Eqs. (30) and (31). Since the derivation closely follows the 3 gluon case, we omit all the details and discuss only the inhomogeneous terms needed at each step. 
Let us start with the colour state $|1234\rangle$. First consider an equation with a zero-order term $D_{20}(1) \equiv D_{20}(1,2+3+4)$. If we want to solve it by $D_{4}^{(1)}=D_{2}(1,2+3+4)$ the three gluons 2,3 and 4 have to combine into a single one with the momentum $k_{2}+k_{3}+k_{4}$. To achieve that we first combine the gluons 2 and 3. According to our prescription explained above, this needs an inhomogeneous term

$$
-(1 / 2) g^{2} N\left(W\left(2,3,4 ; 2^{\prime}, 4^{\prime}\right) \otimes D_{3}^{(1)}\left(1,2^{\prime}, 4^{\prime}\right)+W\left(3,2,1 ; 3^{\prime}, 1^{\prime}\right) \otimes D_{3}^{(1)}\left(1^{\prime}, 3^{\prime}, 4\right)\right)
$$

where $D_{3}^{(1)}(1,2,3)=D_{2}(1,2+3)$. Then we want the gluon $2+3$ to combine with gluon 4 . This will add a new inhomogeneous term

$$
-(1 / 2) g^{2} N\left(W_{2}(2+3,4,1)+W_{2}(4,2+3,1)\right)
$$

With these inhomogeneous terms the solution to the 4 gluon equation can be easily shown to be $D_{4}^{(1)}=D_{3}^{(1)}(1,2+3,4)=D_{2}(1,2+3+4)$. To abbreviate we denote

$$
\begin{aligned}
& W\left(2,3,4 ; 2^{\prime}, 4^{\prime}\right) \otimes D_{3}^{(1)}\left(1,2^{\prime}, 4^{\prime}\right) \equiv W_{3}^{(1)}(2,3,4)=W_{3}^{(1)}(4,3,2) \\
& W\left(3,2,1 ; 3^{\prime}, 1^{\prime}\right) \otimes D_{3}^{(1)}\left(1^{\prime}, 3^{\prime}, 4\right) \equiv W_{3}^{(1)}(3,2,1)=W_{3}^{(1)}(1,2,3)
\end{aligned}
$$

(the superscript (1) denotes the distinguished gluon in the three gluon amplitude: $D_{3}^{(1)}\left(1,2^{\prime}, 4\right)=$ $D_{2}\left(1,2^{\prime}+4\right)$ etc. $)$. With this notation the total inhomogeneous term added for the solution $D_{4}^{(1)}$ is

$$
F_{4}^{(1)}=-(1 / 2) g^{2} N\left(W_{3}^{(1)}(2,3,4)+W_{3}^{(1)}(3,2,1)+W_{2}(2+3,4,1)+W_{2}(4,2+3,1)\right)
$$

Likewise for the solution $D_{4}^{(4)}=D_{2}(4,1+2+3)$ the necessary inhomogeneous term is

$$
F_{4}^{(4)}=-(1 / 2) g^{2} N\left(W_{3}^{(4)}(2,3,4)+W_{3}^{(4)}(3,2,1)+W_{2}(2+3,1,4)+W_{2}(1,2+3,4)\right)
$$

Finally we study the solution $D_{4}^{(14)}=D_{2}(1+4,2+3)$. To construct it we first combine the gluons 23 and afterwards the gluons 14 . The necessary inhomogeneous term is found to be

$$
F_{14}^{(1)}=-(1 / 2) g^{2} N\left(W_{3}^{(2)}(2,3,4)+W_{3}^{(3)}(3,2,1)+W_{2}(4,1,2+3)+W_{2}(1,4,2+3)\right)
$$

Now we combine the solutions $D_{4}^{(1)}, D_{4}^{(4)}$ and $D_{4}^{(14)}$ into the combination (32). The resulting inhomogeneous term is

$$
\begin{aligned}
& \left.\left.F_{4}=-(1 / 8) g^{4} N^{2}\left(W_{3}^{(1)}(2,3,4)\right)+W_{3}^{(4)}(2,3,4)\right)-W_{3}^{(2)}(2,3,4)\right) \\
& \left.+W_{3}^{(1)}(3,2,1)+W_{4}^{(4)}(3,2,1)-W_{3}^{(3)}(3,2,1)+2 W_{2}(1,2+3,4)\right)
\end{aligned}
$$

Compare this with the inhomogeneous terms $D_{2 \rightarrow 4}$ and $D_{3 \rightarrow 4}$ in Eq. (30). The last term in (41) evidently coincides with $D_{2 \rightarrow 4}$. Putting the solution $D_{3}^{(123)}$ into the term $D_{3 \rightarrow 4}$ we find a sum of functions $W_{3}$ which exactly coincides with an analogous sum in (41). Thus we find that (32) is indeed the solution to Eq. (30).

Now we pass to the colour state $|2134\rangle$. Again we first construct four solutions $D_{4}^{(2)}=D_{2}(2,1+$ $3+4), D_{4}^{(3)}=D_{2}(3,1+2+4), D_{4}^{(12)}=D_{2}(1+2,3+4)$ and $D_{4}^{(13)}=D_{2}(1+3,2+4)$. Additional inhomogeneous terms necessary to combine 4 gluons into 2 gluons are found to be (symmetrized in the two possible orders of this procedure)

$$
F_{4}^{(2)}=-(1 / 4) g^{2} N\left(W_{3}^{(2)}(3,4,2)+2 W_{3}^{(2)}(1,3,4)+W_{3}^{(2)}(3,1,2)+\right.
$$




$$
\begin{gathered}
\left.W_{2}(1,3+4,2)+W_{2}(3+4,1,2)+W_{2}(4,1+3,2)+W_{2}(1+3,4,2)\right) \\
F_{4}^{(3)}=-(1 / 4) g^{2} N\left(W_{3}^{(3)}(2,1,3)+2 W_{3}^{(3)}(1,2,4)+W_{3}^{(3)}(2,4,3)+\right. \\
\left.W_{2}(1,2+4,3)+W_{2}(2+4,1,3)+W_{2}(4,1+2,3)+W_{2}(1+2,4,3)\right) \\
F_{4}^{(12)}=-(1 / 4) g^{2} N\left(W_{3}^{(3)}(3,4,2)+W_{3}^{(4)}(1,3,4)+W_{3}^{(1)}(1,2,4)+\right. \\
\left.W_{3}^{(2)}(3,1,2)+W_{2}(3,4,1+2)+W_{2}(3+4,1,2)+W_{2}(4,3,1+2)+W_{2}(1,2,3+4)\right) \\
F_{4}^{(13)}=-(1 / 4) g^{2} N\left(W_{3}^{(2)}(3,4,2)+W_{3}^{(1)}(1,3,4)+W_{3}^{(4)}(1,2,4)+\right. \\
\left.W_{3}^{(3)}(3,1,2)+W_{2}(2,4,1+3)+W_{2}(2+4,1,3)+W_{2}(1,3,2+4)+W_{2}(4,2,1+3)\right)
\end{gathered}
$$

Forming the combination (33) we find the total inhomogeneous term

$$
\begin{gathered}
F_{4}=(1 / 2) g^{2} N\left(F_{4}^{(2)}+F_{4}^{(3)}-F_{4}^{(12)}-F_{4}^{(13)}\right)= \\
-(1 / 16) g^{4} N^{2}\left(2 W_{3}^{(2)}(1,3,4)+2 W_{3}^{(3)}(1,2,4)-W_{3}^{(1)}(1,2,4)-W_{3}^{(1)}(1,3,4)-\right. \\
W_{3}^{(4)}(1,2,4)-W_{3}^{(4)}(1,3,4)+W_{2}(2,1+3,4)+W_{2}(1,3+4,2)+W_{2}(3,1+2,4)+ \\
\left.W_{2}(1,2+4,3)-W_{2}(1+2,3,4)-W_{2}(1,2,3+4)-W_{2}(4,2,1+3)-W_{2}(1,3,2+4)\right)
\end{gathered}
$$

On the other hand, the existing inhomogeneous term (29) can be rewritten using (16) as

$$
\begin{aligned}
D_{3 \rightarrow 4}^{(2134)}=- & (1 / 8) g^{4} N^{2}\left(W_{3}^{(2)}(1,3,4)-W_{3}^{(1)}(1,3,4)-W_{3}^{(4)}(1,3,4)+\right. \\
& \left.W_{3}^{(3)}(1,2,4)-W_{3}^{(1)}(1,2,4)-W_{3}^{(4)}(1,2,4)\right)
\end{aligned}
$$

At first sight they are different, since terms $3 \rightarrow 4$ do not coincide and terms $2 \rightarrow 4$ are absent in (47). However, functions of the type $W_{3}^{(1)}(1,3,4)$ can in fact be expressed in terms of $W_{2}$.

Indeed we have, by definition and using (12),

$$
W_{3}^{(1)}(1,3,4)=\left(V\left(3,1 ; 4^{\prime}-4,1^{\prime}\right)-V\left(3+4,1 ; 4^{\prime}, 1^{\prime}\right)\right) \otimes D_{2}\left(1^{\prime}, 2+4^{\prime}\right)
$$

Shifting the integration variables we have

$$
\begin{gathered}
V\left(3,1 ; 4^{\prime}-4,1^{\prime}\right) \otimes D_{2}\left(1^{\prime}, 2+4^{\prime}\right)=V\left(3,1 ; 3^{\prime}-2-4,1^{\prime}\right) \otimes D_{2}\left(1^{\prime}, 3^{\prime}\right)= \\
W_{2}(2+4,3,1)+V\left(2+3+4,1 ; 3^{\prime}, 1^{\prime}\right) \otimes D_{2}\left(3^{\prime}, 1^{\prime}\right) \\
V\left(3+4,1 ; 4^{\prime}, 1^{\prime}\right) \otimes D_{2}\left(1^{\prime}, 2+4^{\prime}\right)=V\left(3+4,1 ; 2^{\prime}-2,1^{\prime}\right) \otimes D_{2}\left(1^{\prime}, 2^{\prime}\right)= \\
W_{2}(2,3+4,1)+V\left(2+3+4,1 ; 3^{\prime}, 1^{\prime}\right) \otimes D_{2}\left(3^{\prime}, 1^{\prime}\right)
\end{gathered}
$$

Subtracting we get an identity

$$
W_{3}^{(1)}(1,3,4)=W_{2}(2+4,3,1)-W_{2}(2,3+4,1)
$$

and interchanging 1 and 4

$$
W_{3}^{(4)}(1,3,4)=W_{2}(1+2,3,4)-W_{2}(2,1+3,4)
$$

Using these identities we find that all terms with $W_{2}$ in (46) combine into

$$
-W_{3}^{(1)}(1,2,4)-W_{3}^{(1)}(1,3,4)-W_{3}^{(4)}(1,2,4)-W_{3}^{(4)}(1,3,4)
$$

which together with the rest of the terms makes (46) identical to (47).

Thus we have proven that in the leading order in $1 / N$ the solution to the 4 gluon equation is exactly given by (32) and (33) and thus reduces to single BFKL pomerons. 


\section{Next-to-leading order in $1 / N$}

\subsection{Equation for the two-pomeron amplitude}

At next-to-leading order the colour classification of 4 gluon states looses its cyclic symmetry, so that one has to return to the standard description in terms of colour states of the two subsystems of gluons 12 and 34 . We shall be interested in a state which is a color singlet in both subsystems, with a colour wave function

$$
|0\rangle=\left(1 / N^{2}\right) \delta_{a_{1} a_{2}} \delta_{a_{3} a_{4}}
$$

This state is absent in the leading approximation in $1 / N$ and first appears at the next-to-leading order. It is this state that corresponds to the coupling of the projectile with two pomerons and enters the diffractive processes. Projecting the quark loop onto the state (51) we find the corresponding zero-order amplitude:

$$
D_{40}^{(0)}=(1 / 2) g^{2}\left(D_{20}(1)+D_{20}(2)+D_{20}(3)+D_{20}(4)-D_{20}(1+2)-D_{20}(1+3)-D_{20}(1+4)\right)
$$

It is evidently down by a factor $1 / N$ as compared to the leading order amplitudes $D_{4}^{(1234)}$ and $D_{4}^{(2134)}$.

The interaction between gluons in the state $|0\rangle$ may lead them either to the same state (terms with $V_{12}$ and $V_{34}$ ) or to the state in which subsystems 12 and 34 are both in the antisymmetric adjoint representation (the $f$ part of $h$ ). The amplitude for the latter state is already known (in the leading order in $1 / N)$. Therefore transitions from the 4 gluons in the adjoint state into state $|0\rangle$ will serve as an additional inhomogeneous term $D_{4 \rightarrow 4}^{(0)}$ in the equation for the amplitude $D_{4}^{(0)}$. Since no other colour state will enter in the next-to-leading order, the resulting equation for $D_{4}^{(0)}$ will again decouple and have the form

$$
S_{40} D_{4}^{(0)}=D_{40}+D_{2 \rightarrow 4}^{(0)}+D_{3 \rightarrow 4}^{(0)}+D_{4 \rightarrow 4}^{(0)}+g^{2} N\left(V_{12}+V_{34}\right) D_{4}^{(0)}
$$

The zero-order term $D_{4}^{(0)}$ is given by (52). The contributions from the transitions $2 \rightarrow 4$ and $3 \rightarrow 4$ are described by the same diagrams of Fig. 2 and 3, although with new colour factors. One easily finds them to be

$$
D_{2 \rightarrow 4}^{(0)}=-g^{4} N W_{2}(1,2+3,4)
$$

and

$$
\begin{aligned}
D_{3 \rightarrow 4}^{(0)}= & g^{3} \sqrt{2 N}\left(W\left(1,2,3 ; 1^{\prime} 3^{\prime}\right) \otimes D_{3}^{(134)}\left(1^{\prime}, 3^{\prime}, 4\right)-W\left(1,2,4 ; 1^{\prime}, 4^{\prime}\right) \otimes D_{3}^{(134)}\left(1^{\prime}, 3,4^{\prime}\right)+\right. \\
& \left.W\left(2,3,4 ; 2^{\prime}, 4^{\prime}\right) \otimes D_{3}^{(124)}\left(1,2^{\prime}, 4\right)-W\left(1,3,4 ; 1^{\prime}, 4^{\prime}\right) \otimes D_{3}^{(124)}\left(1^{\prime}, 2,4^{\prime}\right)\right)
\end{aligned}
$$

where the amplitude $D_{3}^{(123)}$ is given by (16).

The terms $4 \rightarrow 4$ come from 4 leading colour states $|1234\rangle,|2134\rangle,|4321\rangle$ and $|4312\rangle$ as a result of interactions of gluons belonging to different subsystems, that is upon action of $K_{2 \rightarrow 2}^{(13)}, K_{2 \rightarrow 2}^{(14)}, K_{2 \rightarrow 2}^{(23)}$ and $K_{2 \rightarrow 2}^{(13)}$, where, for example

$$
K_{2 \rightarrow 2}^{(13)}=-g^{2}\left(T_{1} T_{3}\right) V_{13}
$$

Contributions from interactions of gluons across the cylinder (e.g of 1 and 3 in the configuration $|1234\rangle)$ are calculated trivially and we obtain in this manner a part of $D_{4 \rightarrow 4}^{(0)}$

$$
-g^{2}\left(\left(V_{13}+V_{24}\right) D_{4}^{(1234)}+\left(V_{23}+V_{14}\right) D_{4}^{(2134)}\right)
$$


Calculating the contribution from the interaction between adjacent gluons needs some care, since its leading order result is evidently zero. Suppose we want to find $\left\langle 1234\left|K_{2 \rightarrow 2}^{(23)}\right| 0\right\rangle$. For the initial state we can take just as well a cyclic symmetric combination

$$
\left(1 / 2 N^{2}\right)\left(h_{a_{1} a_{2} b} h_{a_{3} a_{4} b}+(2 / N) \delta_{a_{1} a_{2}} \delta_{a_{3} a_{4}}\right)
$$

since all (12)(34) states except the antisymmetric adjoint one give zero. Using the cyclic symmetry we rewrite it as

$$
\left(1 / 2 N^{2}\right)\left(h_{a_{2} a_{3} b} h_{a_{4} a_{1} b}+(2 / N) \delta_{a_{2} a_{3}} \delta_{a_{4} a_{1}}\right)
$$

The first term corresponds to gluons 23 in the adjoint representation, the second - in the colourless one. Acting on this, operator $-\left(T_{2} T_{3}\right)$ gives

$$
\begin{gathered}
\left(1 / 2 N^{2}\right)\left((N / 2) h_{a_{2} a_{3} b} h_{a_{4} a_{1} b}+N(2 / N) \delta_{a_{2} a_{3}} \delta_{a_{4} a_{1}}\right)= \\
(1 / 4 N) h_{a_{1} a_{2} b} h_{a_{3} a_{4} b}+\left(1 / 2 N^{2}\right)\left(\delta_{a_{1} a_{2}} \delta_{a_{3} a_{4}}+\delta_{a_{2} a_{3}} \delta_{a_{4} a_{1}}\right)
\end{gathered}
$$

Projecting onto the state $|0\rangle$ we finally obtain

$$
\left\langle 1234\left|K_{2 \rightarrow 2}^{(23)}\right| 0\right\rangle=(1 / 2) g^{2} V_{23}
$$

An identical contribution comes from the state $|4321\rangle$.

Calculating in this manner all adjacent interactions we find the second half of $D_{4 \rightarrow 4}^{(0)}$;

$$
g^{2}\left(\left(V_{23}+V_{14}\right) D_{4}^{(1234)}+\left(V_{13}+V_{24}\right) D_{4}^{(2134)}\right)
$$

Summing (57) and (58) we finaly find

$$
D_{4 \rightarrow 4}^{(0)}=g^{2}\left(V_{23}+V_{14}-V_{13}-V_{24}\right)\left(D_{4}^{(1234)}-D_{4}^{(2134)}\right)
$$

In the equation (53) the three inhomogeneous terms $D_{2 \rightarrow 4}^{(0)}, D_{3 \rightarrow 4}^{(0)}$ and $D_{4 \rightarrow 4}^{(0)}$ are each the result of some operator applied to the BFKL pomeron, since amplitudes $D_{3}$ and $D_{4}$ entering them are, in fact, linear combinations of BFKL pomerons. Therefore we can present

$$
D_{2 \rightarrow 4}^{(0)}+D_{3 \rightarrow 4}^{(0)}+D_{4 \rightarrow 4}^{(0)}=Z\left(1,2,3,4 ; 1^{\prime} 2^{\prime}\right) \otimes D_{2}\left(1^{\prime}, 2^{\prime}\right)
$$

The kernel $Z$ describes the coupling of the initial pomeron attached to the quark loop (the projectile) to the two final ones. It is our three-pomeron vertex. In the following we shall present a more explicit form of $Z$.

In terms of $Z$ we write the equation for $D_{4}^{(0)}$ as follows

$$
S_{40} D_{4}^{(0)}=D_{20}^{(0)}+Z D_{2}+g^{2} N\left(V_{12}+V_{34}\right) D_{4}^{(0)}
$$

Its solution may evidently be split into two parts: a direct one and the triple pomeron part, which separately satisfy this equation with the inhomogeneous terms $D_{40}^{(0)}$ and $Z D_{2}$, respectively. Both parts can easily be found explicitly due to the fact that the operator acting in (61) is evidently a sum of two independent parts for subsystems 12 and 34 . 


\subsection{The direct contribution}

To solve the equation for the direct part we represent the quark loop diagrams in (1) as a Fourier transform:

$$
g^{2} N f(k)=\int d^{2} r \rho(r) e^{i k r}
$$

Function $\rho(r)$ evidently describes the colour density created by the $q \bar{q}$ pair with a transverse dimension $r$. Using this representation one finds that the term $D_{20}^{(0)}$ (Eq.(52) ) can be represented as

$$
D_{20}^{(0)}=(1 / 4) g^{2} \int d^{2} r \rho(r) \prod_{j=1}^{4}\left(e^{i k_{j} r}-1\right)
$$

It thus factorizes in the gluons at fixed $r$.

Accordingly we can start with an equation for the direct part at fixed $r$ :

$$
S_{40} D_{4}^{(r)}=\prod_{j=1}^{4}\left(e^{i k_{j} r}-1\right)+g^{2} N\left(V_{12}+V_{34}\right) D_{4}^{(r)}
$$

Evidently in this equation subsystems 12 and 34 decouple. The solution to (64) is given by the convolution in the "energy" $1-j$ of two independent BFKL pomerons

$$
D_{4}^{(r)}=\int d j_{12} d j_{34} \delta\left(j-j_{12}-j_{34}\right) D_{2, j_{12}}^{(r)}(1,2) D_{2, j_{34}}^{(r)}(3,4)
$$

Here the pomeron $D_{2, j}^{(r)}(1,2)$ satisfies the equation

$$
S_{20} D_{2, j}^{(r)}=\prod_{j=1}^{2}\left(e^{i k_{j} r}-1\right)+g^{2} N V_{12} D_{2, j}^{(r)}
$$

and similarly for the second pomeron.

The final direct amplitude will result upon integration over $r$ :

$$
D_{4}^{(d i r)}=(1 / 4) g^{2} \int d^{2} r \rho(r) D_{4}^{(r)}
$$

This contribution exactly corresponds to two pomerons directly coupled to the quark loop. As we observe, this coupling factorizes at fixed interquark distance. The corresponding amplitude has thus an eikonal form at fixed $r$. This feature was first established in [4] where it was also generalized to any number of pomerons directly coupled to the quark loop.

\subsection{The three-pomeron vertex}

The triple pomeron part can be written in terms of the Green function of Eq. (61) as

$$
D_{4}^{(\text {triple })}=G_{4}\left(1,2,3,4 ; 1^{\prime}, 2^{\prime}, 3^{\prime}, 4^{\prime}\right) \otimes Z\left(1^{\prime}, 2^{\prime}, 3^{\prime}, 4^{\prime} ; 1^{\prime \prime}, 2^{\prime \prime}\right) \otimes D_{2}\left(1^{\prime \prime}, 2^{\prime \prime}\right)
$$

The Green function $G_{4}$ is evidently a convolution in the energy of two two-gluon (BFKL) Green functions

$$
G_{4, j}\left(1,2,3,4 ; 1^{\prime}, 2^{\prime}, 3^{\prime}, 4^{\prime}\right)=\int d j_{12} d j_{34} \delta\left(j-j_{12}-j_{34}\right) G_{2, j_{12}}\left(1,2, ; 1^{\prime} 2^{\prime}\right) G_{2, j_{34}}\left(3,4 ; 3^{\prime}, 4^{\prime}\right)
$$


If the target part consists of two quark loops, analogous to the projectile loop, then the final solution will be given by two pomerons $D_{2}$ for subsystems 12 and 34 attached to the three-pomeron vertex $Z$ acting on the pomeron $D_{2}$ coming from the projectile.

The three-pomeron vertex $Z$ is implicitly determined by its definition (60), Eqs. (54), (55) and (59) for the terms $D_{2 \rightarrow 4}, D_{3 \rightarrow 4}$ and $D_{4 \rightarrow 4}$ and expressions (16), (32) and (33) for the leading order solutions $D_{3}$ and $D_{4}$. Our aim here is to obtain an explicit expression for $Z$ which will facilitate the study of its infrared behaviour and comparison with the symmetric vertex introduced in [2] for $N=3$.

We start with the term $D_{3 \rightarrow 4}^{(0)}$. Putting (16) into (55) we find terms of two types. Terms of the type $W_{3}^{(3)}(1,2,3)$ can all be reduced to functions $W_{2}$. Taking into account that only terms symmetric under the interchange 1 and 2 and/or 3 and 4 contribute, we find for this part

$$
D_{3 \rightarrow 4}^{(01)}=(1 / 2) g^{4} N\left(W_{2}(1+4,2,3)+W(1+3,2,4)-2 W(3,1+2,4)+(1234 \rightarrow 4321)\right.
$$

The second part of $D_{3 \rightarrow 4}^{(0)}$ is formed by terms of the type $W_{3}^{(1)}(2,3,4)$. Using (12) and the bootstrap relation we find

$$
(1 / 2) g^{2} N W_{3}^{(1)}(2,3,4)=D_{2}(1)(\omega(2+3)+\omega(3+4)-\omega(2+3+4)-\omega(3))
$$

As a result, the second part of $D_{3 \rightarrow 4}^{(0)}$ turns out to be

$$
\begin{aligned}
D_{3 \rightarrow 4}^{(02)} & =-g^{2} D_{2}(1)(\omega(2+3)+\omega(3+4)-\omega(2+3+4)-\omega(3)) \\
& -(1234 \rightarrow 2134)-(1234 \rightarrow 3124)-(1234 \rightarrow 4123)
\end{aligned}
$$

To further simplify these expressions and also compare them with [2] we express functions $W_{2}$ for the total momentum zero via the function $G\left(k_{1}, k_{2}\right)=G\left(k_{2}, k_{1}\right)$ introduced in [2]. For $1+2+3=0$

$$
g^{2} N W_{2}(1,2,3)=G(1+2,2+3)-D_{2}(1+2)(\omega(2)-\omega(1+2))-D_{2}(2+3)(\omega(2)-\omega(2+3))
$$

In terms of functions $G$, summing (70) and (72) we find

$$
\begin{gathered}
D_{3 \rightarrow 4}^{(0)}=(1 / 2) g^{2}(G(1,2+4)+G(2,1+4)+G(3,1+4)+G(4,1+3)-2 G(1,2)-2 G(3,4)- \\
D_{2}(1+3)(\omega(2)+\omega(3)-2 \omega(1+3))-D_{2}(1+4)(\omega(2)+\omega(3)-2 \omega(1+4))+ \\
D_{2}(1)(\omega(1)+\omega(3)-2 \omega(1+4))+D_{2}(2)(\omega(2)+\omega(3)-2 \omega(1+3))+ \\
\left.D_{2}(3)(\omega(2)+\omega(3)-2 \omega(1+3))+D_{2}(1)(\omega(2)+\omega(4)-2 \omega(1+4))\right)
\end{gathered}
$$

This expression is not symmetric in gluons 12 nor in gluons 34 . However the function $D_{4}^{(0)}$ is symmetric in both gluon pairs. So we should symmetrize (74) in gluon pairs 12 and 34 . The resulting symmetric expression is

$$
\begin{gathered}
D_{3 \rightarrow 4}^{(0)}=(1 / 4) g^{2}(G(1,2+3)+G(1,2+4)+G(2,1+3)+G(2,1+4) \\
+G(3,1+4)+G(3,2+4)+G(4,1+3)+G(4,2+3)-4 G(1,2)-4 G(3,4)- \\
D_{2}(1+3)\left(\sum_{i=1}^{4} \omega(i)-4 \omega(1+3)\right)-D_{2}(1+4)\left(\sum_{i=1}^{4} \omega(i)-4 \omega(1+4)\right)+ \\
D_{2}(1)(2 \omega(1)+\omega(3)+\omega(4)-2 \omega(1+3)-2 \omega(1+4))+D_{2}(2)(2 \omega(2)+\omega(3)+\omega(4)-2 \omega(1+3)-2 \omega(1+4))+
\end{gathered}
$$




$$
\left.D_{2}(3)(2 \omega(3)+\omega(1)+\omega(2)-2 \omega(1+3)-2 \omega(1+4))+D_{2}(1)(2 \omega(4)+\omega(1)+\omega(2)-2 \omega(1+3)-2 \omega(1+4))\right)
$$

Note that in this notation the term $D_{2 \rightarrow 4}^{(0)}$ can be rewritten as

$$
D_{2 \rightarrow 4}^{(0)}=-g^{2}\left(G(1,4)+D_{2}(1)(\omega(1)-\omega(2+3))+D_{2}(4)(\omega(4)-\omega(2+3))\right)
$$

After symmetrization in 12 and 34 it becomes

$$
\begin{gathered}
D_{2 \rightarrow 4}^{(0)}=-(1 / 4) g^{2}(G(1,3)+G(1,4)+G(2,3)+G(2,4)+ \\
D_{2}(1)(2 \omega(1)-\omega(1+3)-\omega(1+4))+D_{2}(2)(2 \omega(2)-\omega(1+3)-\omega(1+4))+ \\
\left.D_{2}(3)(2 \omega(3)-\omega(1+3)-\omega(1+4))+D_{2}(4)(2 \omega(4)-\omega(1+3)-\omega(1+4))\right)
\end{gathered}
$$

We finally come to the term $D_{4 \rightarrow 4}^{(0)}$. We put expressions (32) and (33) for $D_{4}^{(1234)}$ and $D_{4}^{(2134)}$ into (59). Operator $V_{23}+V_{14}-V_{13}-V_{24}$ is antisymmetric under the interchange of 1 and 2 and/or 3 and 4. Since the two-pomeron state is symmetric under these substitutions, only antisymmetric parts of $D_{4}^{(1234)}$ and $D_{4}^{(2134)}$ give a nonzero contribution. Taking this into account we find

$$
D_{4 \rightarrow 4}^{(0)}=(1 / 4) g^{4} N\left(V_{23}+V_{14}-V_{13}-V_{24}\right)\left(D_{2}(1+3)-D_{2}(1+4)\right)
$$

Here we again meet with two differnt types of terms: with the interaction between different gluons $\left(\right.$ e.g $\left.V_{13} D_{2}(1+4)\right)$ or within the same gluon (e.g. $\left.V_{13} D_{2}(1+3)\right)$.

The first type leads to a contribution

$$
D_{4 \rightarrow 4}^{(01)}=(1 / 4) g^{4} N\left(\left(V_{13}+V_{24}\right) D_{2}(1+4)+\left(V_{23}+V_{14}\right) D_{2}(1+3)\right)
$$

To reduce it to the expression of the same type as before we use an identity, which is proven in the Appendix

$$
V_{13} D_{2}(1+4)=W_{2}(2+3,1,4)+W_{2}(2,3,1+4)-W_{2}(2,1+3,4)-W_{2}(2+3,0,1+4)
$$

Introducing then functions $G$ we find for this part

$$
\begin{gathered}
D_{4 \rightarrow 4}^{(01)}=(1 / 4) g^{2}(G(1,2+3)+G(1,2+4)+G(2,1+3)+ \\
G(2,1+4)+G(3,1+4)+G(3,2+4)+G(4,1+3)+G(4,2+3)- \\
G(1,3)-G(1,4)-G(2,3)-G(2,4)-2 G(2+3,1+4)-2 G(1+3,2+4)+ \\
D_{2}(1)(\omega(2+3)+\omega(2+4)-\omega(3)-\omega(4))+D_{2}(2)(\omega(1+3)+\omega(1+4)-\omega(3)-\omega(4))+ \\
D_{2}(3)(\omega(2+4)+\omega(1+4)-\omega(1)-\omega(2))+D_{2}(4)(\omega(1+3)+\omega(2+3)-\omega(1)-\omega(2))- \\
\left.\left(D_{2}(1+3)+D_{2}(1+4)\right) \sum_{i=1}^{4} \omega(i)\right)
\end{gathered}
$$

The second part of the contribution is

$$
\begin{gathered}
D_{4 \rightarrow 4}^{(02)}=-(1 / 4) g^{4} N\left(\left(V_{13}+V_{24}\right) D_{2}(1+3)+\right. \\
\left.\left(V_{23}+V_{14}\right) D_{2}(1+4)\right)
\end{gathered}
$$


It is much simpler. Application of the bootstrap relation immediately gives

$$
D_{4 \rightarrow 4}^{(02)}=(1 / 2) g^{2} N\left(D_{2}(1+4)\left(\sum_{i=1}^{4} \omega(i)-2 \omega(1+4)\right)+D_{2}(1+3)\left(\sum_{i=1}^{4} \omega(i)-2 \omega(1+3)\right)\right)
$$

The final three-pomeron vertex is obtained after summing (75), (77), (81) and (83). It is remarkable that all terms involving functions $D_{2}(i)$ or $D_{2}(i+k)$ cancel and the resulting expression contains only functions $G$ :

$$
\begin{gathered}
Z D_{2}=(1 / 2) g^{2}(G(1,2+3)+G(1,2+4)+G(2,1+3)+ \\
G(2,1+4)+G(3,1+4)+G(3,2+4)+G(4,1+3)+G(4,2+3)- \\
2 G(1,2)-2 G(3,4)-G(1,3)-G(1,4)-G(2,3)-G(2,4)-G(2+3,1+4)-G(1+3,2+4))
\end{gathered}
$$

The vertex $Z$ itself can be easily extracted from this expression separating the operator which acts on $D_{2}$.

One immediately observes that the found vertex is infrared stable. It is expressed via functions $G$, which are explicitly infrared stable by construction (see [2]). Moreover one easily finds from (84) that the vertex $Z$ goes to zero if any of its external variables (1,2,3 or 4 in (84)) goes to zero. This means that it can be safely integrated with two pomerons $D_{2}(1,2)$ and $D_{2}(3,4)$ which describe its coupling to the two target colourless particles. By construction it is symmetric in gluons 12 and 34 and under the interchange $12 \leftrightarrow 34$. Thus it satisfies all requirements relevant from the physical point of view. However, as one observes from (84), it is not fully symmetric in all the gluons as the transitional vertex constructed in [2]. Related to this, it contains somewhat less terms than in [2] (16 instead of 19) and with different coefficients. We shall discuss the physical reason for this difference in the next section.

\section{Discussion}

The study of the 4 -gluon system at $N \rightarrow \infty$ leads to a very simple and physically expected picture. In the leading approximation the system reduces to a single BFKL pomeron, the 4 gluons in the colour configuration of a cylinder coalescing into a pair of gluons. In the next-to-leading order the diffractive amplitude appears, which clearly divides into a direct contribution (double pomeron exchange), with the two pomerons directly coupled to the quark loop, and a triple pomeron contribution, with a single pomeron splitting into two pomerons by a three-pomeron vertex.

The found three-pomeron vertex is different from the one originally constructed by J.Bartels and M.Wuesthoff in [2]. The reason is not related to the limit $N \rightarrow \infty$, as such, but to a different treatment of the 4- gluon amplitude in the colour state $|0\rangle$. In [2] this amplitude was supposed to also contain a contribution from 2-gluon states (BFKL pomerons) in the form of the reggeized zero-order term (52). As a result in Eq. (53) new terms $4 \rightarrow 4$ appear which desribe transitions from the reggeizing to non-reggeizing pieces in the same colour state $|0\rangle$. This naturally changes the transitional vertex and explains the difference between between our vertex and that of [2].

However, in our opinion, the assumption about the existence of a reggeizing piece in the 4 gluon system in the state $|0\rangle$ lacks any foundation. Reggeization, say, of the term $D_{20}(1+2)$ in (52) 
implies the existence of a colourless reggeon, identical to the physical one. Reggeization of the term $D_{20}(1+3)$ implies the existence of such a reggeon in the symmetric representation of the highest dimension. These possibilities are not supported by the BFKL equation for different colour states. The $N \rightarrow \infty$ approach definitely rejects them. In it the zero-order term (52) does not reggeize into a single pomeron but rather into two pomerons, thus leading to a well-defined and intuitively expected double pomeron exchange contribution. For these reasons we consider our three-pomeron vertex (84) better founded and suited to the decription of the diffractive amplitude, as the original one in [2].

\section{Acknowledgments}

The author expresses his deep gratitude to Prof. C.Pajares, who attracted his attention to the problem, and to Prof. J.Bartels for illuminating discussions, which served as a starting point for this study.

\section{Appendix. Transformation of the term $V_{13} D_{2}(1+4)$}

The term of interest is explicitly written as

$V\left(1,3 ; 1^{\prime}, 3^{\prime}\right) \otimes D_{2}\left(1^{\prime}+4,2+3^{\prime}\right)=V\left(1,3 ; 1^{\prime}-4,3^{\prime}-2\right) \otimes D_{2}\left(1^{\prime}, 2\right)=V\left(1,3 ; 4^{\prime}-4,-4^{\prime}-2\right) \otimes D_{2}\left(4^{\prime},-4^{\prime}\right)$

In the last equality we used the conservation law $1+2+3+4=0$. The identity (12) can be rewritten, again, with the conservation law taken into account, as

$$
W\left(4,1,3 ; 4^{\prime}, 1^{\prime}\right)=V\left(1,3 ; 4^{\prime}-4,1+3+4-4^{\prime}\right)-V\left(1+4,3 ; 4^{\prime}, 1+3+4-4^{\prime}\right)
$$

Since $1+3+4=-2$, integrating (86) with $D_{2}\left(4^{\prime},-4^{\prime}\right)$ and comparing with (85) we find

$$
V_{13} D_{2}(1+4)=\left(W\left(4,1,3 ; 4^{\prime}, 1+3+4-4^{\prime}\right)+V\left(1+4,3 ; 4^{\prime}, 1+3+4-4^{\prime}\right)\right) \otimes D_{2}\left(4^{\prime},-4^{\prime}\right)
$$

Consider the two terms on the right-hand side separately. Interchanging 3 and 4 in the first we find $\left(3^{\prime}=1+3+4-4^{\prime}\right)$ :

$$
\begin{gathered}
W\left(3,1,4: 3^{\prime}, 4^{\prime}\right) \otimes D_{2}\left(4^{\prime}, 3^{\prime}-1-3-4\right)=\left(V\left(1,4 ; 3^{\prime}-3,4^{\prime}\right)\right. \\
\left.-V\left(1+3,4,3^{\prime}, 4^{\prime}\right)\right) \otimes D_{2}\left(4^{\prime}, 3^{\prime}-1-3-4\right)=\left(V\left(1,4 ; 3^{\prime \prime}+1+4,4^{\prime}\right)-V\left(1+3,4,3^{\prime \prime}+1+3+4,4^{\prime}\right)\right) \otimes D_{2}\left(4^{\prime}, 3^{\prime \prime}\right)
\end{gathered}
$$

where $3 "=3$ '-1-3-4. Both terms can be expressed via $W$, using (12). We obtain

$$
\begin{gathered}
W\left(-1-4,1,4: 3^{\prime}, 4^{\prime}\right)+V\left(-4,4 ; 3^{\prime} 4^{\prime}\right)-W\left(-1-3-4,1+3,4 ; 3^{\prime}, 4^{\prime}\right) \\
\left.-V\left(-4,4 ; 3^{\prime}, 4^{\prime}\right)\right) \otimes D_{2}\left(4^{\prime}, 3^{\prime}\right)=W_{2}(-1-4,1,4)-W_{2}(-1-3-4,1+3,4)
\end{gathered}
$$

The second term in (87) is transformed analogously

$$
\begin{gathered}
V\left(3,1=4 ; 3^{\prime}, 4^{\prime}\right) \otimes D_{2}\left(4^{\prime}, 3^{\prime}-1-3-4\right)=V\left(3,1+4 ; 3^{\prime \prime}+1+3+4,4^{\prime}\right) \otimes D_{2}\left(4^{\prime}, 3^{\prime \prime}\right)= \\
W_{2}(-1-3-4,3,1+4)+V\left(-1-4,1+4 ; 3^{\prime}, 4^{\prime}\right) \otimes D_{2}\left(4^{\prime}, 3^{\prime}\right)
\end{gathered}
$$


Next we take into account that $-1-4=2+3,-1-3-4=2$ and that

$$
V\left(2+3,1+4 ; 3^{\prime}, 4^{\prime}\right) \otimes D_{2}\left(4^{\prime}, 3^{\prime}\right)=-W_{2}(2+3,0,1+4)
$$

Taking the sum of (89) and (90 we then obtain (80). 


\section{References}

1. J.Bartels, Z.Phys.C60 (1993) 471.

2. J.Bartels and M.Wuesthoff, Z.Phys. C66 (1995) 157.

3. J.Bartels, L.N.Lipatov and M.Wuesthoff, Nucl. Phys. B 464 (1996) 298.

4. M.A.Braun, Z.Phys. C71 (1996) 601.

5. L.N.Lipatov in: "Perturbative QCD", ed. A.H.Mueller, Advanced Series in High Energy Physics (World Scientific, Singapore, 1989).

6. J.Bartels, Nucl. Phys. B175 (1980)365.

7. L.N.Lipatov, Yad. Fiz. bf 23 (1976) 642.

\section{Figure captions}

Fig. 1. The transition from 2 to 3 gluons.

Fig. 2. The transition from 2 to 4 gluons.

Fig. 3. The transition from 3 to 4 gluons. 


\section{Figures}

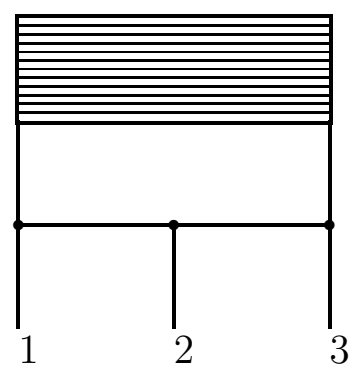

Fig. 1

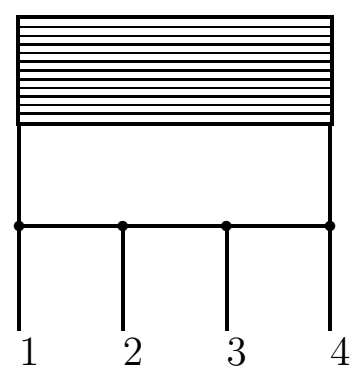

Fig. 2 

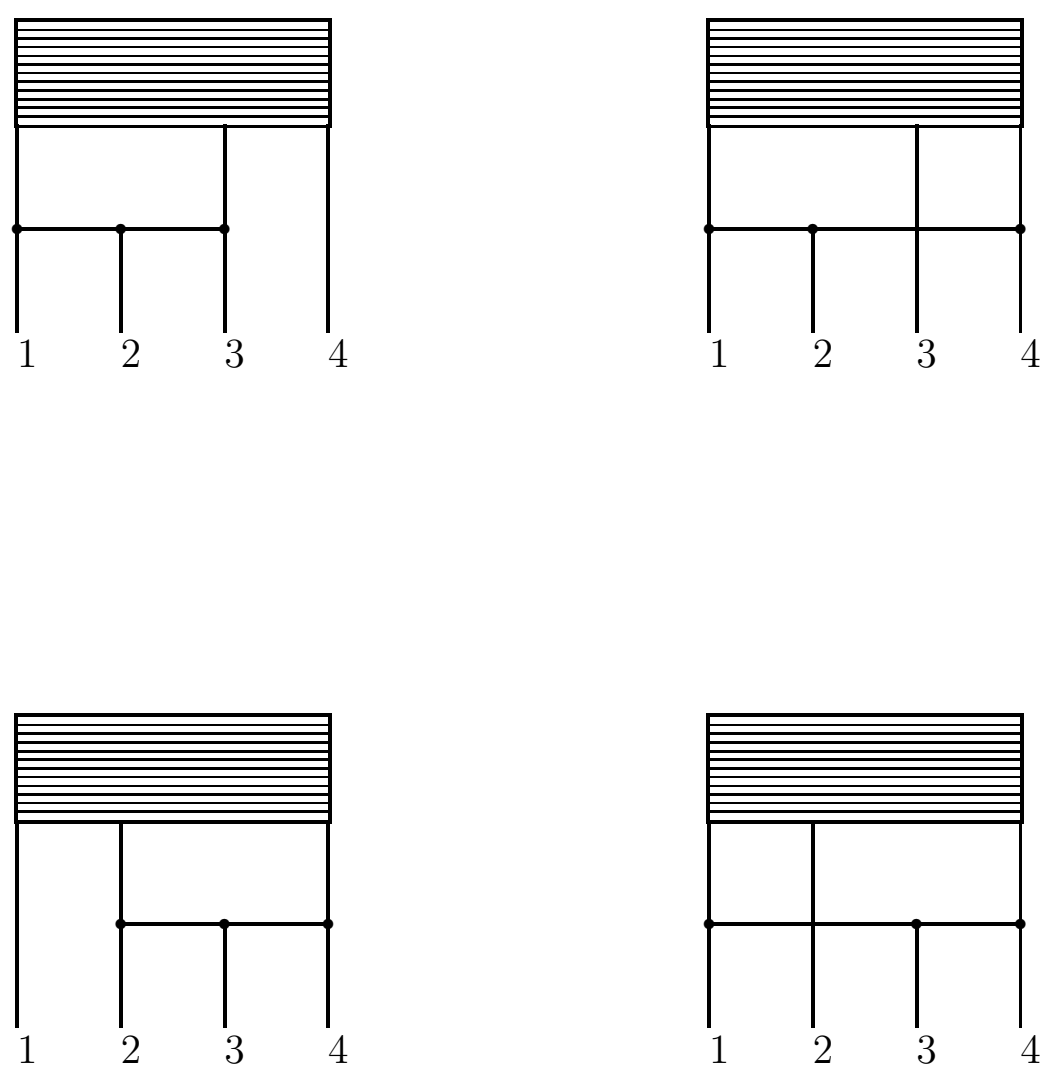

Fig.3 